\title{
SYMPTOMATIC PROXIMAL FIBULAR SWELLINGS IN SIBLINGS WITH HEREDITARY MULTIPLE EXOSTOSES MANAGED BY MODIFIED RESECTION TECHNIQUE
}

\author{
Raju Prabhakar Singh1, Sekar Prabhakar ${ }^{2}$
}

${ }^{1}$ Senior Assistant Professor, Department of Orthopaedics, Govt. Kilpauk Medical College and Hospital, Chennai. ${ }^{2}$ Assistant Professor, Department of Orthopaedics, Govt. Kilpauk Medical College and Hospital, Chennai.

\section{ABSTRACT}

Osteochondromas are the most common tumour of proximal fibula. Resection of the tumour is fraught with complications due to proximity of neurovascular structures and ligaments. We present a case report of two cases of hereditary multiple exostoses in siblings with symptomatic proximal fibula exostoses, which were managed by modified resection technique for proximal fibula tumours. The benefit of this technique is preservation of proximal tibiofibular joint and its associated ligaments. One case developed transient common peroneal nerve palsy, which completely recovered in the follow-up period. Both patients at one year follow-up had full range of motion of knee without any motor weakness and neurodeficit and without any signs or symptoms of instability of knee joint or recurrence.

\section{KEYWORDS}

Hereditary Multiple Exostoses, Proximal Fibula Osteochondroma, Malawer Type I.

HOW TO CITE THIS ARTICLE: Singh RP, Prabhakar S. Symptomatic proximal fibular swellings in siblings with hereditary multiple exostoses managed by modified resection technique. J. Evolution Med. Dent. Sci. 2016;5(42):2643-2646, DOI: $10.14260 /$ jemds/2016/618

\section{INTRODUCTION}

Fibular tumours comprise $2.5 \%$ of total primary bone tumours.[1] The most common tumours of this location are osteochondroma, osteosarcoma, Ewing's sarcoma, giant cell tumour. Eccentric growth of the osteochondromas as a result of solitary osteochondroma or hereditary multiple exostoses may lead to a variety of symptoms. Proximal fibular osteochondromas due to their close vicinity to the neurovascular bundle can cause compressive neuropathy of the peroneal nerve.[2] or vascular compression syndrome and pseudoaneurysm of popliteal vessels.[3] Depending upon the extent of the growth, surgical treatment of proximal fibular osteochondromas may vary from debulking.[4] to resection of proximal fibula. ${ }^{[5]}$ However, after resection of proximal fibula, it is necessary to repair lateral collateral ligament and biceps femoris to prevent lateral laxity of knee.

\section{MATERIALS AND METHODS}

Two sisters, one 17-year-old and another 14-year-old suffering from multiple bony swellings in different parts of the body around major joints presented in our hospital in October 2015. The swellings appeared insidiously and gradually grew in size and became symptomatic. Patient's father, elder brother and elder sister had similar complaints in the past. Patients were diagnosed with hereditary multiple exostoses. There was no neurovascular deficit or restriction of joint mobility. Patient complained of pain and cosmetic deformity due to the lesions. AP and lateral view radiograph and MRI of affected extremities were taken. Hence, patients were planned to undergo modified fibular resection for symptomatic proximal fibular osteochondroma as described by

Financial or Other, Competing Interest: None.

Submission 05-04-2016, Peer Review 02-05-2016,

Acceptance 07-05-2016, Published 26-05-2016.

Corresponding Author:

Dr. Raju Prabhakar Singh,

148 C, First Floor,

Mithra Beauty Spot Apartment,

Aani Street, Chinmaya Nagar

Virugambakkam,

Chennai-600092.

E-mail: rpsingh.msortho@gmail.com

DOI: $10.14260 /$ jemds $/ 2016 / 618$
Mootha AK et al[6] and extraperiosteal excision of remaining symptomatic osteochondromas in October 2015.

\section{SURGICAL TECHNIQUE}

All cases were operated under tourniquet in lateral decubitus position. Incision was made starting approximately at $8 \mathrm{~cm}$ proximal to the midpoint of the popliteal skin crease and extended to the head of the fibula and then straightened and further extended along the line of fibular shaft approximately $5 \mathrm{~cm}$ below the planned level of osteotomy. The flaps were raised anteriorly till $2-3 \mathrm{~cm}$ lateral to the anterior tibial crest and posteriorly till the midline. Once adequately exposed, common peroneal nerve was explored first. It was easily palpated near the fibular head, as it passes around the inferior border of the biceps muscle. The peroneal nerve was approached with the intent of mobilizing the common peroneal nerve and opening and exposing the common peroneal and deep peroneal nerve branch throughout the fibromuscular tunnel as described by Ryan et al.[7] This was necessary to retract the peroneal nerve safely to a more anterolateral position.

It is imperative to ensure complete mobilization and release of the narrow part of the peroneal nerve through the fibrous tunnel to prevent postoperative compression on the nerve due to reactive swelling. During this process, it may be essential to sacrifice recurrent genicular branches of the peroneal nerve. After this, the dissection was proceeded distally at the level of the planned fibular osteotomy. This was calculated from preoperative X-rays taking a free margin of 3$5 \mathrm{~cm}$ from the inferior most extent of the stalk of the tumour from fibular shaft. After elevation of periosteum, a transverse osteotomy was done with an oscillating saw. From the level of osteotomy, the anterior and lateral compartment muscles were elevated subperiosteally till their origins from the proximal fibula. The dissection stops anteriorly once the interosseous membrane is reached and posteriorly once the aponeurosis separating the peronei from the posterior flexors was reached.

To visualize the extent of the osteochondroma, we need to dissect further posteriorly. Lateral gastrocnemius was mobilized and retracted towards midline exposing the soleus 
proximally and Flexor Hallucis Longus (FHL) distally. Soleus and FHL are released from fibula leaving a cuff of these muscles attached to the fibula (extraperiosteal release). No attempt was made to expose the posterior neurovascular bundle. An osteotomy was done through the head of the fibula at level of physis in case of immature skeleton or just distal to the PTFJ in case of mature skeleton and this level was confirmed under fluoroscopy. At this time, care was taken not to open the PTFJ, thus leaving the capsule and ligaments of the joint undisturbed. Once this osteotomy was completed, the fibular fragment along with the osteochondromatous growth is free except for the interosseous septum.

The osteotomised fibular fragment was held with a bone holder and internally rotated, thus exposing the neurovascular bundle. The neurovascular bundle was carefully raised off the interosseous septum and then the interosseous septum was divided delivering out the fibular fragment along with the tumorous growth. Closure was done under negative suction drain.

Postoperatively, a long leg splint was applied for 1 week and then strengthening and mobilization exercises were started.

\section{CASE 1}
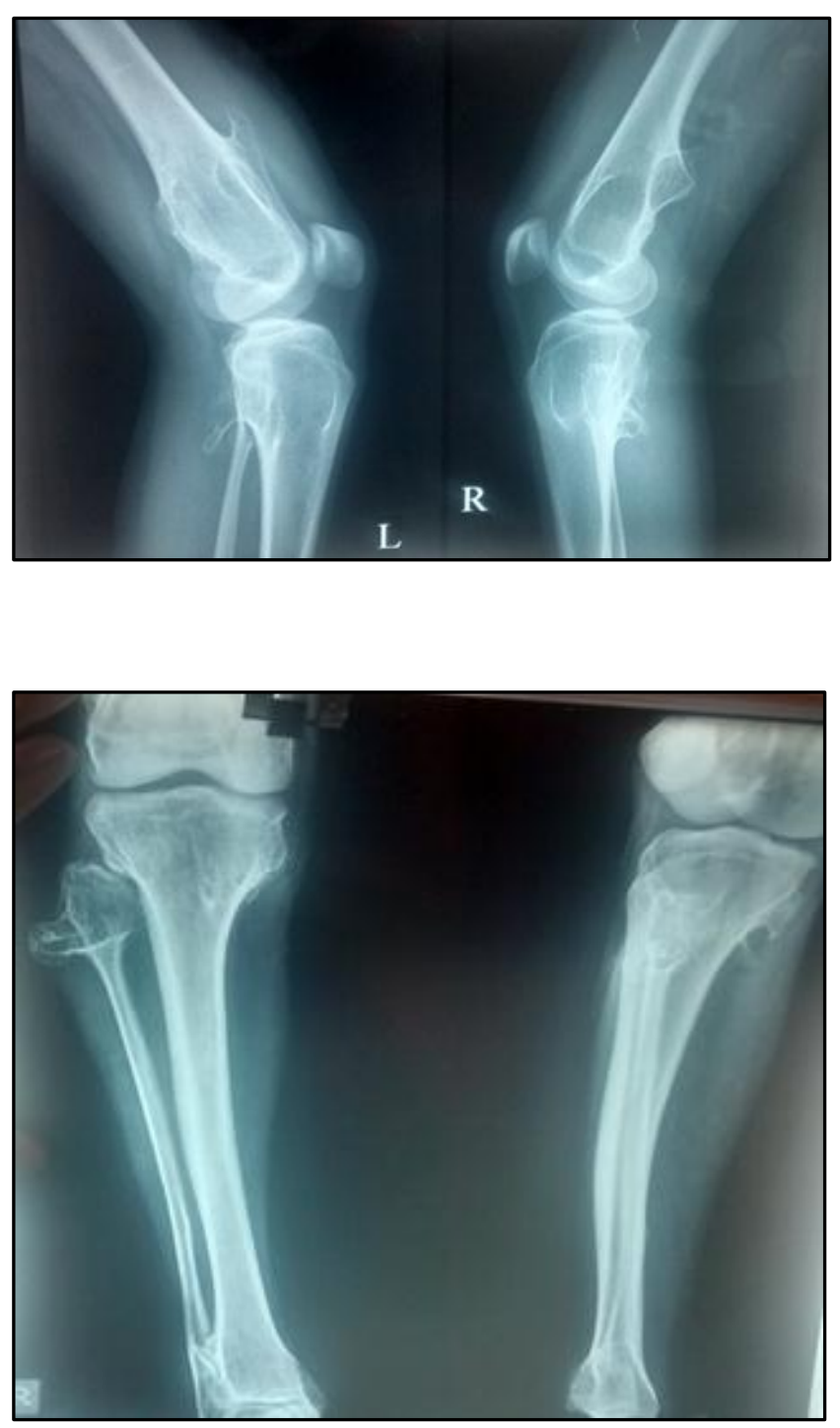

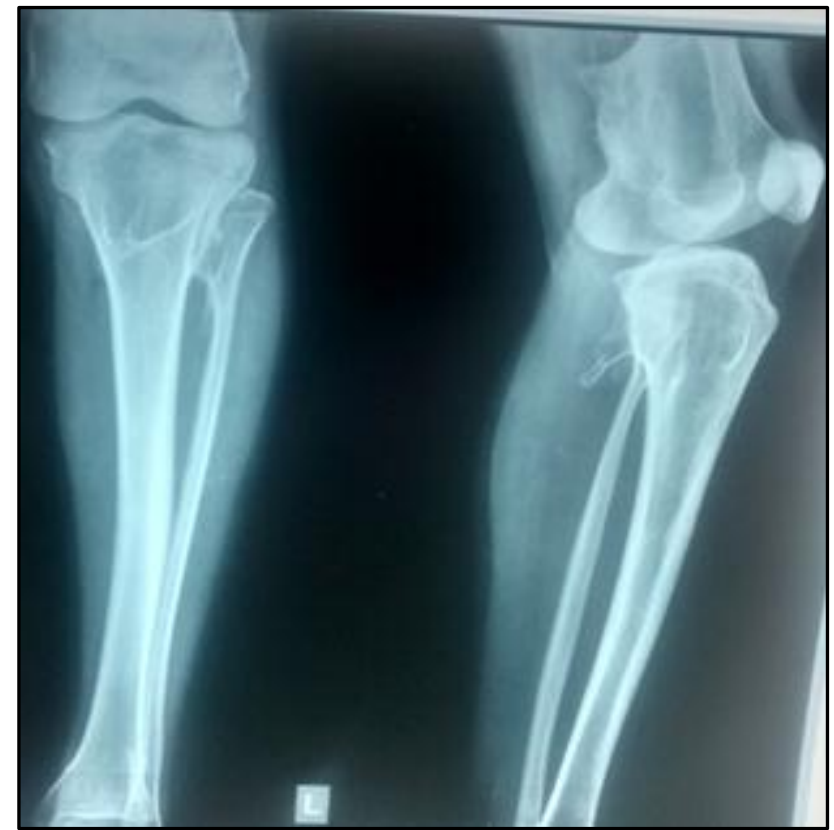

\section{INTRAOPERATIVE PICS}

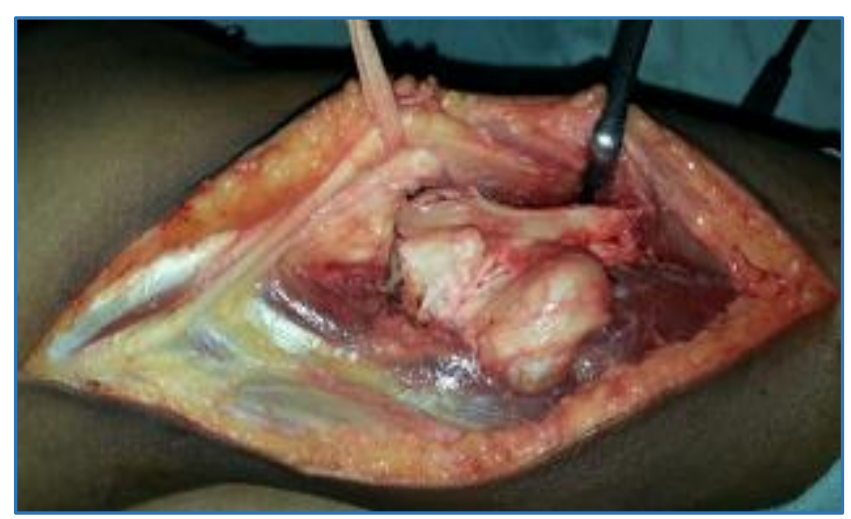

\section{POSTOPERATIVE X-RAY}

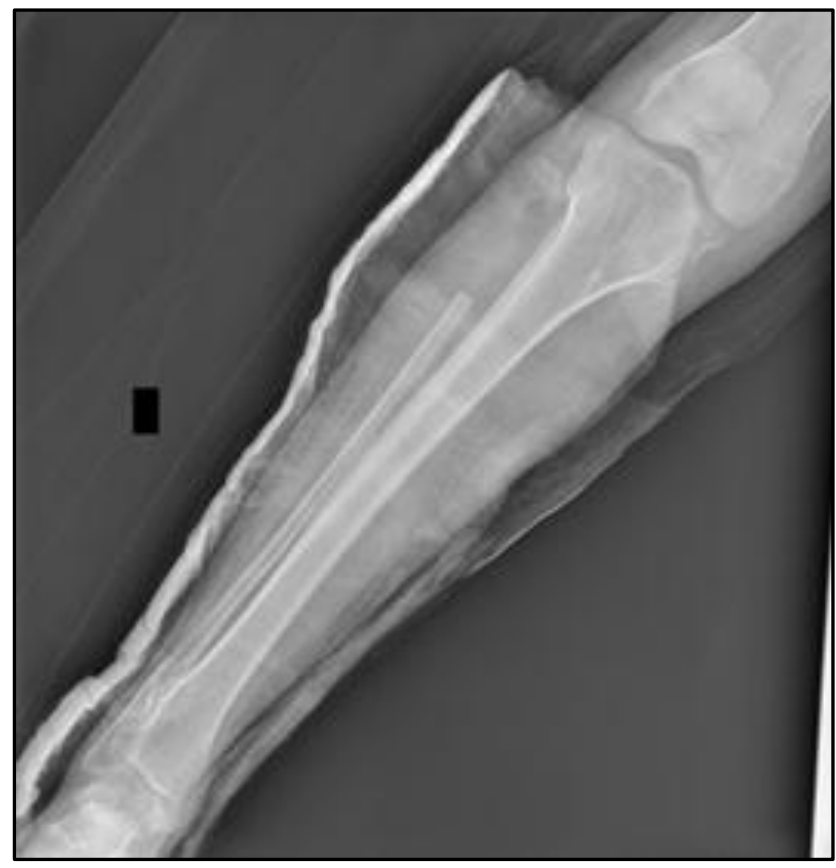




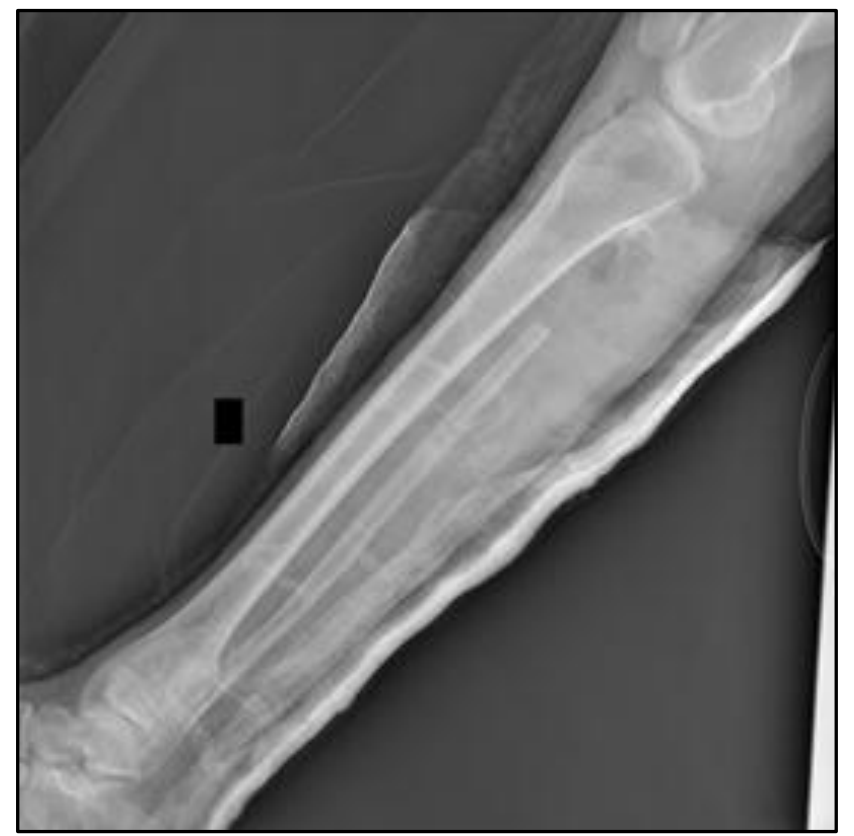

\section{CASE 2}
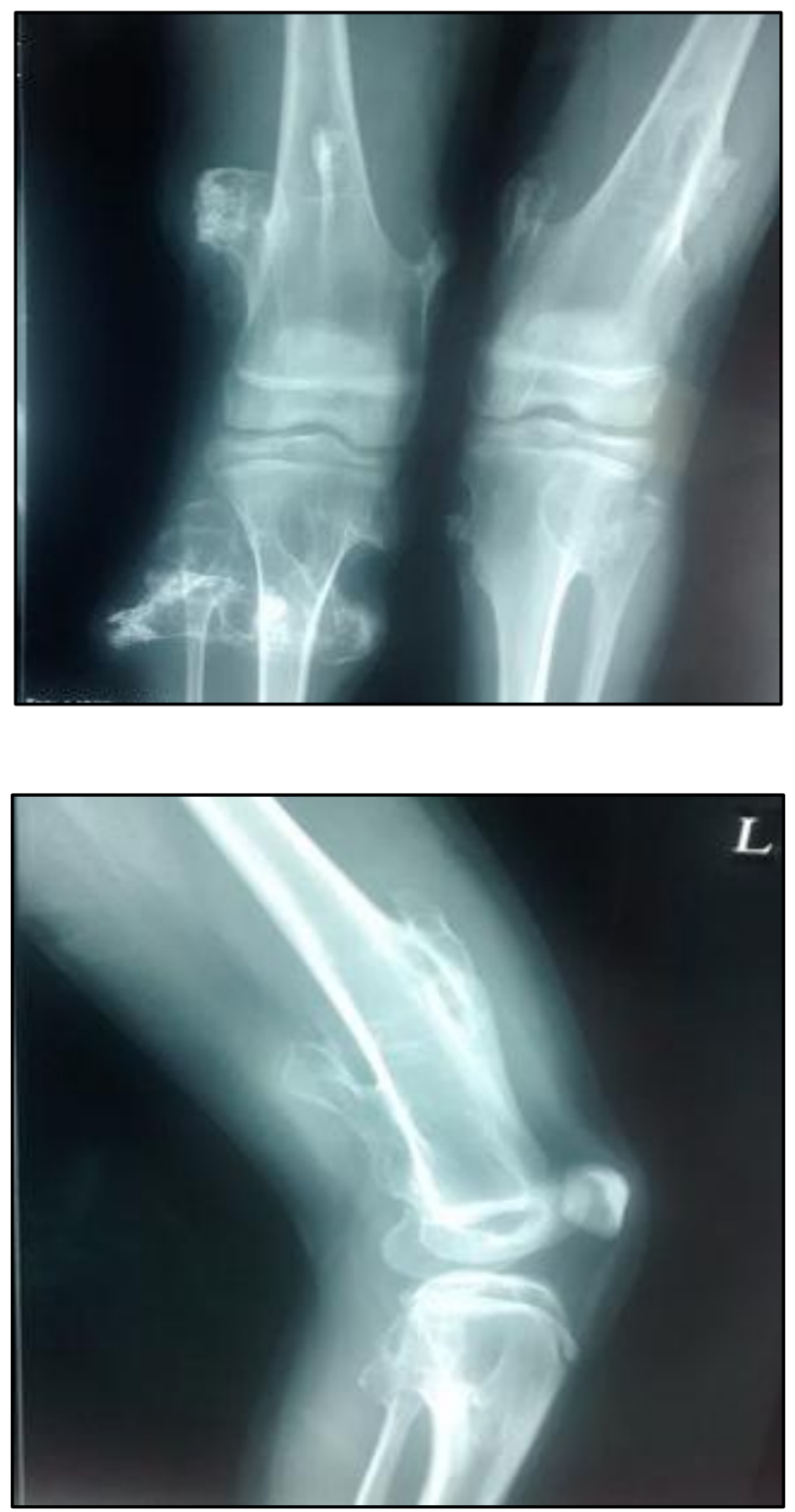

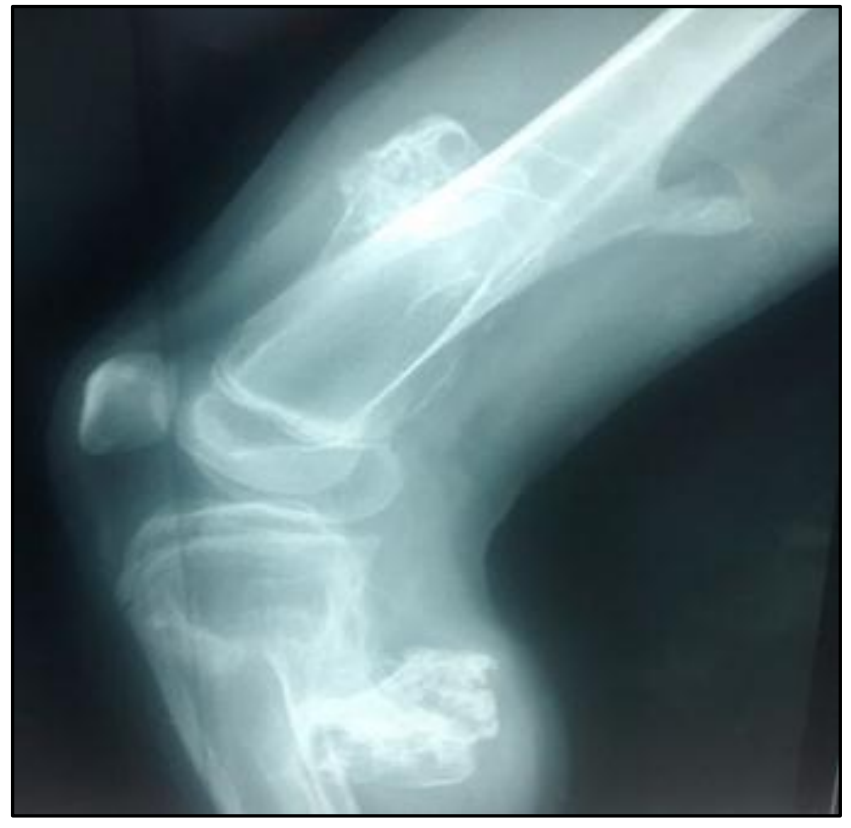

\section{INTRAOPERATIVE PICS}
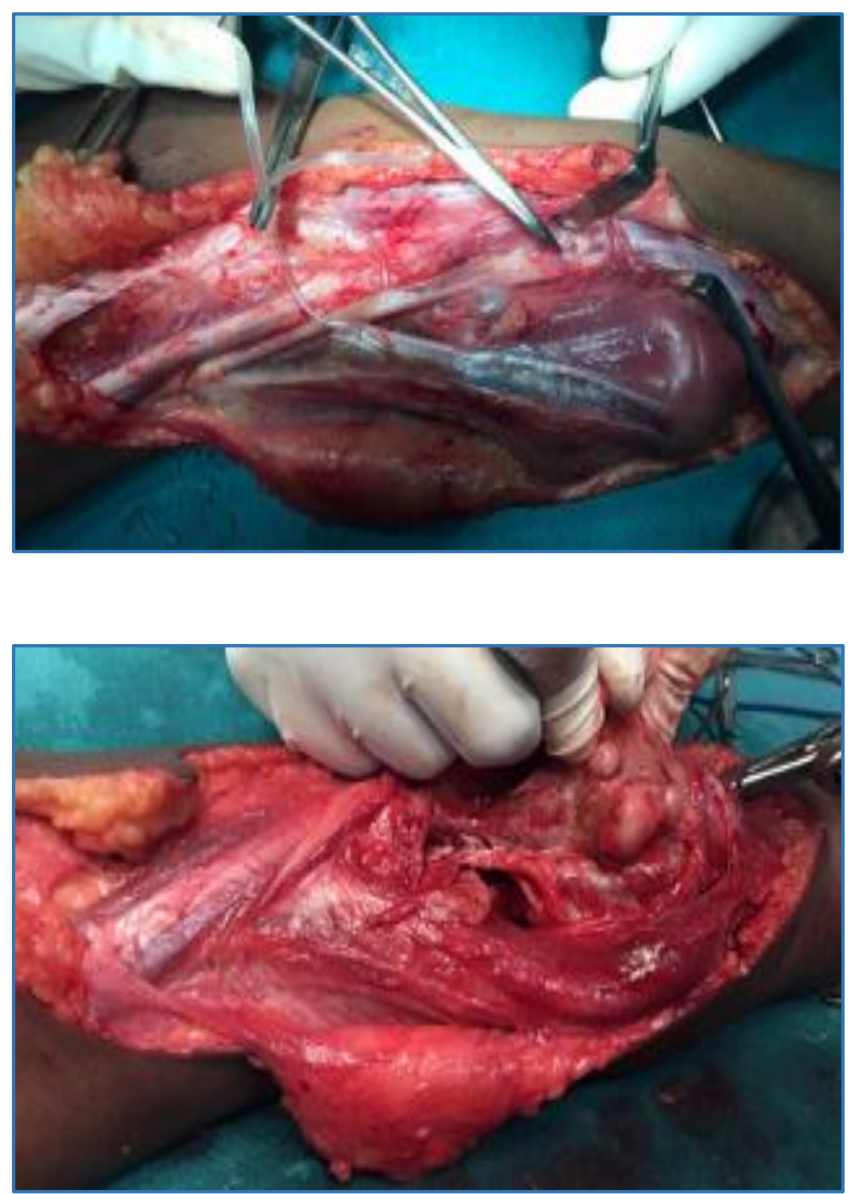


\section{POSTOP X-RAY}
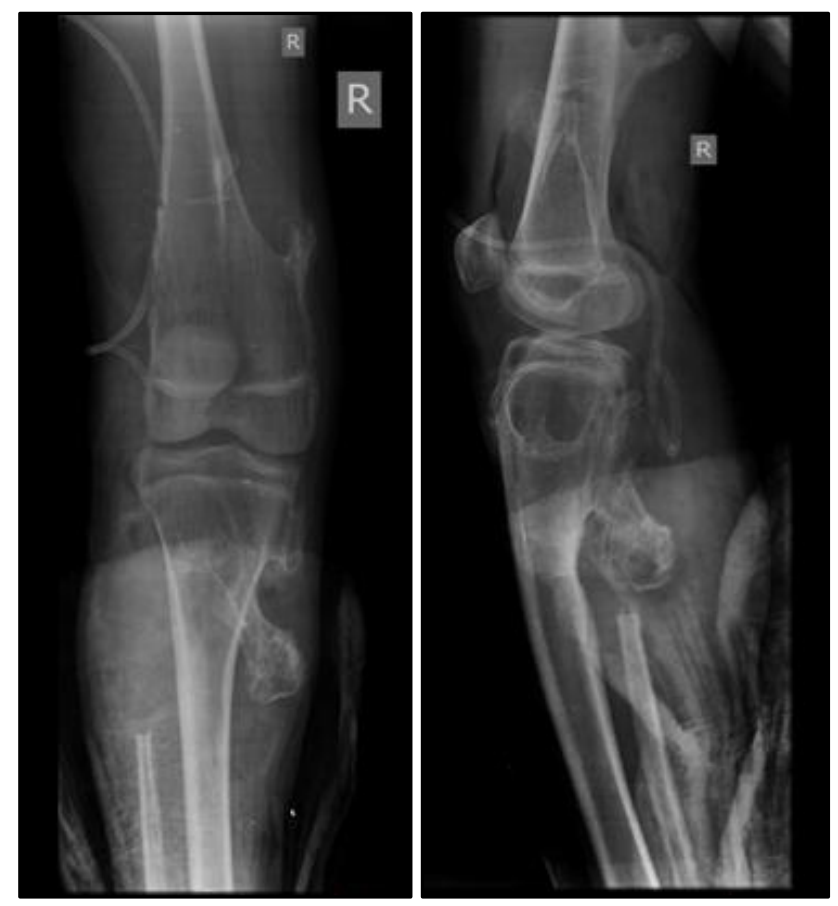

\section{RESULTS}

One patient had transient common peroneal nerve palsy, which gradually improved. Both patients at six-month followup had full range of motion of knee without any motor weakness and neurodeficit. There were no signs of instability of knee joint or recurrence at six-month follow-up.

\section{DISCUSSION}

Surgery for proximal fibular osteochondromas vary from simple debulking to complete excision of proximal fibula (Malawer Type I resection).[5] Many a time, it is virtually impossible to approach the stalk of the osteochondromatous growth and thus requires excision of the whole proximal fibula. ${ }^{[5,8]}$ Modified fibular resection technique preserves the PTFJ and at the same time the whole osteochondromatous growth is removed en masse with shaft of fibula. This technique also differs from conventional Malawer Type I proximal fibular resection.[2] that most of the dissection is subperiosteal.

By utilizing modified resection technique PTFJ, lateral stabilizing structures of knee were preserved. This technique enabled complete resection of tumour with good functional outcome.

\section{REFERENCES}

1. Dahlin DC. Bone tumors, general aspects and data on 6221 cases. Springfield, Charles C Thomas, with figure. 1978;11-12.

2. Cardelia JM, Dormans JP, Drummond DS, et al. Proximal fibular osteochondroma with associated peroneal nerve palsy: a review of six cases. J Paediatr Orthop 1995;15(5):574-7.

3. Guy NJ, Shetty AA, Gibb PA. Popliteal artery entrapment syndrome: an unusual presentation of a fibular osteochondroma. Knee 2004;11(6):497-9.

4. Mnif H, Koubaa M, Zrig M, et al. Peroneal nerve palsy resulting from fibular head osteochondroma. Orthopaedics 2009;32(7):528.

5. Malawer MM. Surgical management of aggressive and malignant tumours of the proximal fibula. Clin Orthop Relat Res 1984;186:172-81.

6. Mootha AK, Saini R, Dhillon M, et al. Modified resection technique for proximal fibular osteochondromas. Orthop Traumatol Surg Res 2011;97(5):569-73.

7. Ryan W, Mahony N, Delaney M, et al. Relationship of the common peroneal nerve and its branches to the head and neck of the fibula. Clin Anat 2003;16(6):501-5.

8. Gray KV, Robinson J, Bernstein RM, et al. Splitting of common peroneal nerve by an osteochondroma: two case reports. J Paediatr Orthop B 2004;13(4):281-3. 